\title{
Risk of endometrial cancer in relation to individual nutrients from diet and supplements
}

\author{
Rita K Biel ${ }^{1, *}$, llona Csizmadi ${ }^{1,2,3}$, Linda S Cook ${ }^{1,2,4}$, Kerry S Courneya ${ }^{5}$, \\ Anthony M Magliocco ${ }^{1,3,6}$ and Christine M Friedenreich ${ }^{1,2,3}$ \\ 'Division of Cancer Care, Department of Population Health Research, Alberta Health Services, \\ 1331 - 29 St. N.W., Calgary, Alberta T2N 4N2, Canada: ${ }^{2}$ Department of Community Health Sciences, \\ Faculty of Medicine, University of Calgary, Calgary, Alberta, Canada: ${ }^{3}$ Department of Oncology, Faculty \\ of Medicine, University of Calgary, Calgary, Alberta, Canada: ${ }^{4}$ Department of Internal Medicine, NM Health \\ Sciences Center, University of New Mexico, Albuquerque, NM, USA: ${ }^{5}$ Faculty of Physical Education \\ and Recreation, E488 Van Vliet Centre, University of Alberta, Edmonton, Alberta, Canada: ${ }^{6}$ Department of \\ Pathology and Laboratory Medicine, Faculty of Medicine, University of Calgary, Calgary, Alberta, Canada
}

Submitted 15 December 2010: Accepted 4 April 2011: First published online 14 July 2011

\begin{abstract}
Objective: Intake of nutrients may influence the risk of endometrial cancer (EC). We aimed to estimate the association of intake of individual nutrients from food and from food plus supplements with EC occurrence.

Design: A population-based case-control study conducted in Canada (2002-2006). Setting: Nutrient intakes from food and supplements were assessed using an FFQ. Logistic regression was used to estimate EC risk within quartile levels of nutrient intakes.

Subjects: Incident EC cases ( $n$ 506) were identified from the Alberta Cancer Registry, and population controls were frequency- and age-matched to cases ( $n$ 981).

Results: There existed little evidence of an association with EC for the majority of macronutrients and micronutrients examined. We observed a statistically significant increased risk associated with the highest, compared with the lowest, quartile of intake of dietary cholesterol (multivariable-adjusted OR $=1 \cdot 51,95 \%$ CI $1 \cdot 08,2 \cdot 11$; $P$ for trend $=0 \cdot 02$ ). Age-adjusted risk at the highest level of intake was significantly reduced for $\mathrm{Ca}$ from food sources $(\mathrm{OR}=0 \cdot 73,95 \% \mathrm{CI} 0 \cdot 54,0 \cdot 99)$ but was attenuated in the multivariable model $(\mathrm{OR}=0 \cdot 82,95 \% \mathrm{CI} 0 \cdot 59,1 \cdot 13)$. When intake from supplements was included in $\mathrm{Ca}$ intake, risk was significantly reduced by $28 \%$ with higher Ca (multivariable-adjusted OR $=0 \cdot 72,95 \% \mathrm{CI} 0 \cdot 51,0 \cdot 99, P$ for trend $=0 \cdot 04$ ). We also observed unexpected increased risks at limited levels of intakes of dietary soluble fibre, vitamin $\mathrm{C}$, thiamin, vitamin $\mathrm{B}_{6}$ and lutein/zeaxanthin, with no evidence for linear trend.

Conclusions: The results of our study suggest a positive association between dietary cholesterol and EC risk and an inverse association with Ca intake from food sources and from food plus supplements.
\end{abstract}

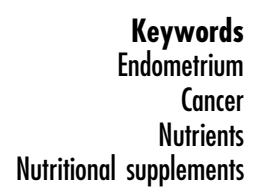

Keywords

Cancer

utrients

Nutritional supplements
Endometrial cancer (EC) ranks as the fourth most common cancer among women in Canada, with an estimated 4500 women newly diagnosed and 790 estimated deaths from this cancer in $2010^{(1)}$. Obesity and oestrogen-only menopausal hormone therapy (HT) expose women to higher levels of oestrogen and are well-established risk factors for $\mathrm{EC}^{(2)}$. Diet may also mediate endogenous oestrogen levels, promoting endometrial carcinogenesis $^{(2)}$. A 2007 review concluded that the evidence for an association between diet and EC was 'limited', but 'suggestive' for a decrease in risk with consumption of nonstarchy vegetables, particularly cruciferous vegetables, and for an increase in risk with consumption of red meat, providing rationale for further study of diet in EC aetiology ${ }^{(3)}$. A conclusive link with macro- and micronutrients has not been established. Micronutrient intake from food alone may not fully reflect an individual's overall biological micronutrient dose, as vitamins and minerals are also widely consumed as supplements. The prevalence of vitamin/mineral supplement use in Canada was recently estimated to be $47 \%$ among women, and higher $60 \%$ among women aged $\geq 51$ years ${ }^{(4)}$. Hence, misclassification among exposure levels has likely impacted the observed dietary associations with EC, contributing to inconsistent findings among studies. Few studies have accounted for these additional nutrient sources through 
adjustment, through addition to diet or by restriction to nonusers of supplements. Only two studies have examined EC risk in association with micronutrient intake from food and supplements combined, examining a limited number of micronutrients $^{(5,6)}$. To address this gap, we collected information on a wide range of micronutrients from food and supplements through a case-control study of EC.

\section{Methods}

\section{Study population}

A population-based case-control study of EC risk was conducted in Alberta, Canada, and details of this study have been reported $^{(7)}$. As part of the present study, information was collected on past-year dietary intake. All participants provided signed informed consent, and ethical approval was obtained from the Alberta Cancer Board and from the University of Calgary. Cases, identified from the Alberta Cancer Registry, comprised women aged 30-79 years with incident, primary-site EC diagnosed in Alberta between 12 September 2002 and 15 February 2006. Controls comprised $\geq 30$-year-old Alberta residents recruited through random digit dialling and frequency-matched to cases 2:1 on the basis of age $( \pm 5 \text { years })^{(8)}$. All participants completed in-person interviews and a self-administered diet history questionnaire (DHQ). The participation rate was $67.9 \%$ ( $n$ 549) for cases and 52.2\% ( $n$ 1036) for controls. We excluded eleven women because of unsatisfactory interviews, forty-seven women because they did not complete a DHQ and forty women because they reported total daily energy intake values $<2510 \mathrm{~kJ}(600 \mathrm{kcal}) / \mathrm{d}(n$ 33) and $>20920 \mathrm{~kJ}(5000 \mathrm{kcal}) / \mathrm{d}(n$ 7). Thus, 506 cases and 981 controls were included in the present analysis.

\section{Data collection}

In-person interviews were conducted and anthropometric measurements were taken. Participants self-reported their past-year dietary intake using a Canadian version of the National Cancer Institute's (NCI) $\mathrm{DHQ}^{(9)}$ following their interview. The cognitively tested DHQ queries frequency and portion size intakes of 124 individual food items. A subset of questions ascertains seasonal food intake, special foods and use or addition of fat. The original US version was validated against biomarkers of energy expenditure and protein intake ${ }^{(10-12)}$. Because of differences in nutrient fortification ${ }^{(13)}$, the Canadian DHQ nutrient database was previously modified for use in Canada ${ }^{(14)}$. The 'Diet*Calc' Analysis Program version $1 \cdot 4 \cdot 3$ (National Cancer Institute, Bethesda, MD, USA) was used to derive nutrient intake variables.

Twenty-seven nutrients were examined in total. Macronutrients included total energy $(\mathrm{kJ}(\mathrm{kcal}))$, protein $(\mathrm{g})$, total fat $(\mathrm{g})$, saturated fat $(\mathrm{g})$, monounsaturated fat $(\mathrm{g})$, polyunsaturated fat $(\mathrm{g})$, discretionary fat $(\mathrm{g})$, cholesterol (mg), carbohydrate (g), dietary fibre NDSR (Nutrition
Data Systems for Research; g), insoluble fibre (g) and soluble fibre $(\mathrm{g})$. We examined food and supplement intakes, expressed as daily intakes, of twelve individual micronutrients including vitamin A (retinol equivalents), $\beta$-carotene $(\mu \mathrm{g})$, vitamin C $(\mathrm{mg})$, vitamin D $(\mu \mathrm{g})$, vitamin $\mathrm{E}$ (mg $\alpha$-tocopherol equivalents), folate ( $\mu$ g dietary folate equivalents (DFE)), thiamin $\left(\mathrm{B}_{1} ; \mathrm{mg}\right)$, riboflavin $\left(\mathrm{B}_{2} ; \mathrm{mg}\right)$, niacin $(\mathrm{mg})$, vitamin $\mathrm{B}_{6}(\mathrm{mg})$, vitamin $\mathrm{B}_{12}(\mu \mathrm{g})$ and $\mathrm{Ca}$ (mg). Methionine (g) and additional phytochemicals lycopene $(\mu \mathrm{g})$ and lutein/zeaxanthin $(\mu \mathrm{g})$ were examined from food sources only. Total folate intake, expressed as DFE, was estimated by accounting for the greater bioavailability of synthetic folic acid compared with naturally occurring food folate ${ }^{(15)}$. Synthetic folic acid was multiplied by 1.7 before this amount was added to naturally occurring food folate to derive total DFE from food or from food and supplements ${ }^{(15,16)}$.

\section{Statistical analyses}

Nutrients were natural log transformed and adjusted for total energy using the residual method ${ }^{(17)}$. Unconditional logistic regression analysis was used to estimate OR and 95\% CI for the risk of EC within nutrient intake quartiles according to the distribution among controls in age-adjusted and multivariable-adjusted models. If an association with EC was detected, possible effect modification was assessed by stratifying these models by BMI $\left(<25 \cdot 0 v\right.$. $\left.\geq 25 \cdot 0 \mathrm{~kg} / \mathrm{m}^{2}\right)$, waist circumference $(<88 \quad v$. $\geq 88 \mathrm{~cm})$ and menopausal status (pre/perimenopausal $v$. postmenopausal) and by examining the interaction terms between nutrient intake exposure levels and these effect modifiers in logistic models. A stratified analysis by HT use was also undertaken to examine postmenopausal women not taking HT compared with those taking only oestrogen plus progesterone $(\mathrm{E}+\mathrm{P}) \mathrm{HT}$. The following factors were considered as confounders because of their known or suspected associations with both EC risk and diet: age (years), total energy intake (kJ (kcal)/d; as per the residuals-adjusted model, except in the case of modelling total energy intake as an exposure), age at menarche (years), BMI $\left(<25 \cdot 0\right.$ v. $\left.25 \cdot 0-29 \cdot 9, \geq 30 \cdot 0 \mathrm{~kg} / \mathrm{m}^{2}\right)$, parity ( $0 v$. $1-2$, more than two pregnancies at $\geq 20$ weeks' gestation), education (lower than high school $v$. high school or higher), hypertension history (ever $v$. never), history of type 2 diabetes (ever $v$. never), hormone contraceptive use (never $v$. ever), HT use combined with menopausal status (post/perimenopausal/no HT $v$. post/perimenopausal + oestrogen; post/perimenopausal $+\mathrm{E}+\mathrm{P} ;$ post/perimenopausal + other menopausal hormones; premenopausal), lifetime alcohol consumption ( 0 drinks $v .<1$ drink, $\geq 1$ drink/d) and smoking (smoker, ex-smoker or current smoker). All covariates were first individually assessed.

Age and total energy intake were included in all models. Nutrient-specific supplement use (yes $v$. no) was included in all micronutrient diet models, except for lycopene, lutein/zeaxanthin and methionine. Covariates were deleted from saturated models using a backward 
elimination procedure ${ }^{(18)}$, with the exception of biologically important covariates. A significant change in effect was defined as $>10 \%$ difference in the point estimate from the saturated model OR. Fully adjusted models included age, total energy intake, age at menarche, BMI, parity, education, hypertension history, hormone contraception use and HT use combined with menopausal status. Model fit was assessed using the Hosmer-Lemeshow goodnessof-fit test ${ }^{(19)}$. Tests for linear trend were conducted for all models of categorized data, with an ordinal-score variable treated as a continuous variable and all $P$ values reported as two-sided. All statistical analyses were performed using the STATA statistical software package version $10 \cdot 0$ (StataCorp LP, College Station, TX, USA) ${ }^{(51)}$.

\section{Results}

Our study population consisted primarily of Caucasian (96\%), postmenopausal (74\%) women, 28-79 years of

Table 1 Characteristics of endometrial cancer cases and controls and age-adjusted OR for risk of endometrial cancer with non-dietary risk factors, Alberta, Canada, 2002-2006 (n 1487)

\begin{tabular}{|c|c|c|c|c|c|c|}
\hline \multirow[b]{2}{*}{ Characteristic } & \multicolumn{2}{|c|}{ Cases (n 506) } & \multicolumn{2}{|c|}{ Controls ( $n$ 981) } & \multirow[b]{2}{*}{ Age-adjusted OR } & \multirow[b]{2}{*}{$95 \% \mathrm{Cl}$} \\
\hline & Mean or $n$ & SD or $\%$ & Mean or $n$ & SD or $\%$ & & \\
\hline Age (years) & $58 \cdot 7$ & $9 \cdot 2$ & $58 \cdot 3$ & $10 \cdot 1$ & - & - \\
\hline BMI $\left(\mathrm{kg} / \mathrm{m}^{2}\right)^{*}$ & $32 \cdot 3$ & $7 \cdot 9$ & $28 \cdot 1$ & $5 \cdot 7$ & - & - \\
\hline$<25 \cdot 0$ & 92 & 18 & 321 & 33 & $1 \cdot 00$ & - \\
\hline $25 \cdot 0-29 \cdot 9$ & 136 & 27 & 357 & 36 & $1 \cdot 32$ & $0.98,1.80$ \\
\hline$\geq 30 \cdot 0$ & 278 & 55 & 302 & 31 & $3 \cdot 20$ & $2 \cdot 41,4 \cdot 25$ \\
\hline Waist circumference $(\mathrm{cm}) \dagger$ & $97 \cdot 9$ & $18 \cdot 7$ & $87 \cdot 5$ & $14 \cdot 6$ & - & - \\
\hline$<88$ & 163 & 32 & 550 & 57 & $1 \cdot 00$ & - \\
\hline$\geq 88$ & 339 & 68 & 422 & 43 & $2 \cdot 71$ & $2 \cdot 16,3 \cdot 39$ \\
\hline Age at menarche (years) $\ddagger$ & $12 \cdot 3$ & $1 \cdot 5$ & $12 \cdot 6$ & 1.5 & $0 \cdot 89$ & $0.83,0.96$ \\
\hline Parity (pregnancies at $\geq 20$ weeks' gestation) & $2 \cdot 2$ & $1 \cdot 5$ & $2 \cdot 6$ & $1 \cdot 6$ & - & - \\
\hline 0 & 89 & 18 & 101 & 10 & $1 \cdot 00$ & - \\
\hline $1-2$ & 220 & 43 & 411 & 42 & 0.58 & $0 \cdot 42,0 \cdot 81$ \\
\hline$>2$ & 197 & 40 & 469 & 48 & 0.42 & $0.30,0.60$ \\
\hline \multicolumn{7}{|l|}{ HT use by menopausal status $\S \|$} \\
\hline Post/perimenopausal - no HT & 283 & 56 & 487 & 50 & $1 \cdot 00$ & - \\
\hline Post/perimenopausal - E only & 19 & 4 & 23 & 2 & $1 \cdot 37$ & $0.73,2.58$ \\
\hline Post/perimenopausal - E + P & 125 & 25 & 322 & 35 & 0.66 & $0.51,0.85$ \\
\hline Post/perimenopausal - other HT & 26 & 5 & 26 & 3 & $1 \cdot 73$ & $0.98,3.03$ \\
\hline Premenopausal & 52 & 10 & 116 & 12 & $0 \cdot 85$ & $0.55,1.30$ \\
\hline Hormone contraception use (ever) & 308 & 61 & 695 & 71 & 0.63 & $0.50,0.80$ \\
\hline Hypertension (ever) & 213 & 42 & 257 & 26 & $2 \cdot 08$ & $1 \cdot 65,2 \cdot 63$ \\
\hline Type 2 diabetes (ever) ${ }^{\star *}$ & 60 & 12 & 56 & 6 & $2 \cdot 19$ & $1 \cdot 49,3 \cdot 22$ \\
\hline \multicolumn{7}{|l|}{ Educational leveltt } \\
\hline High school or lower & 167 & 33 & 273 & 28 & $1 \cdot 00$ & - \\
\hline Above high school & 339 & 67 & 707 & 72 & $0 \cdot 79$ & $0.63,1.00$ \\
\hline Total physical activity (MET $\times \mathrm{h} /$ week per year) & $119 \cdot 2$ & $32 \cdot 4$ & $120 \cdot 1$ & $32 \cdot 7$ & - & - \\
\hline$\leq 96 \cdot 47$ & 123 & 24 & 246 & 25 & $1 \cdot 00$ & - \\
\hline$>96 \cdot 47$ to $\leq 117 \cdot 75$ & 141 & 28 & 245 & 25 & $1 \cdot 13$ & $0.83,1.53$ \\
\hline$>117 \cdot 75$ to $\leq 139 \cdot 90$ & 125 & 25 & 245 & 25 & $1 \cdot 00$ & $0 \cdot 74,1 \cdot 36$ \\
\hline$>139 \cdot 90$ to $\leq 275 \cdot 43$ & 117 & 23 & 245 & 25 & 0.92 & $0 \cdot 67,1 \cdot 27$ \\
\hline \multicolumn{7}{|l|}{ Smoking } \\
\hline Never/fewer than 100 cigarettes & 257 & 51 & 489 & 50 & $1 \cdot 00$ & - \\
\hline Ex-smoker & 194 & 38 & 369 & 38 & $1 \cdot 00$ & $0.79,1 \cdot 25$ \\
\hline Current smoker & 55 & 11 & 123 & 12 & $0 \cdot 86$ & $0.60,1 \cdot 22$ \\
\hline \multicolumn{7}{|l|}{ 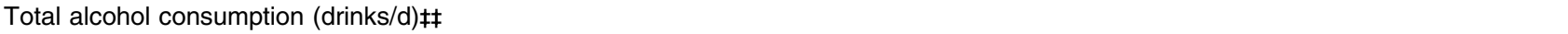 } \\
\hline 0 (non-drinkers) & 105 & 21 & 149 & 15 & $1 \cdot 00$ & - \\
\hline$\leq 1$ & 387 & 76 & 804 & 82 & 0.69 & $0.52,0.91$ \\
\hline$>1$ & 14 & 3 & 28 & 3 & 0.72 & $0.36,1 \cdot 44$ \\
\hline \multicolumn{7}{|l|}{ Supplement use (multivitamin or nutrient-only type) } \\
\hline No & 81 & 16 & 120 & 12 & $1 \cdot 00$ & - \\
\hline Yes & 425 & 84 & 861 & 88 & $0 \cdot 72$ & $0.53,0.98$ \\
\hline
\end{tabular}

$\mathrm{HT}$, hormone therapy; $\mathrm{E}$, oestrogen; $\mathrm{E}+\mathrm{P}$, oestrogen + progesterone; MET, metabolic equivalents.

*Height and weight self-reported for five cases and five controls, not reported by one control.

tNot reported for four cases and nine controls.

¥Not reported for two cases.

$\S \mathrm{HT}$ use not reported for one case and six controls.

IMenopausal status not reported in one case and one control.

-Not reported in two controls; OR compared with never.

${ }^{\star *}$ Not reported in three controls; OR compared with never.

t+Not reported in one control.

¥¥Lifetime total alcohol consumption (minimum of six drinks per year) assessed at the time of interview. Participants reported the number of drinks of beer ( $1 \mathrm{can} / \mathrm{glass} / \mathrm{bottle}=12 \mathrm{oz} / 360 \mathrm{ml})$, wine $(1 \mathrm{glass}=5 \mathrm{oz} / 140 \mathrm{ml})$ or liquor $(1.5 \mathrm{oz} / 45 \mathrm{ml})$ consumed per week for each pattern of drinking. Responses were used to estimate alcohol consumption in grams of ethanol per year, and this estimate was converted to number of drinks per day. 
Table 2 Daily nutrient intakes reported on the DHQ by cases and controls

\begin{tabular}{|c|c|c|c|c|}
\hline \multirow[b]{2}{*}{ Daily intake } & \multicolumn{2}{|c|}{ Cases $(n 506)$} & \multicolumn{2}{|c|}{ Controls ( $n$ 981) } \\
\hline & Mean & SD & Mean & SD \\
\hline \multicolumn{5}{|l|}{ Macronutrients } \\
\hline Total energy $(\mathrm{kJ})^{\star}$ & $6730 \cdot 4$ & $2626 \cdot 7$ & $6682 \cdot 3$ & $2620 \cdot 4$ \\
\hline Protein $(\mathrm{g})$ & $64 \cdot 9$ & $25 \cdot 6$ & $64 \cdot 8$ & $28 \cdot 0$ \\
\hline Total fat $(g)$ & $60 \cdot 2$ & $30 \cdot 0$ & $58 \cdot 8$ & $29 \cdot 1$ \\
\hline Saturated fat (q) & $19 \cdot 3$ & $9 \cdot 8$ & $19 \cdot 0$ & $10 \cdot 0$ \\
\hline Monounsaturated fat $(\mathrm{g})$ & $22 \cdot 6$ & 11.9 & $22 \cdot 0$ & $11 \cdot 3$ \\
\hline Polyunsaturated fat $(\mathrm{g})$ & $13 \cdot 6$ & $7 \cdot 5$ & $13 \cdot 2$ & $7 \cdot 2$ \\
\hline Discretionary fat $(\mathrm{g})$ & $47 \cdot 0$ & $25 \cdot 0$ & $46 \cdot \overline{0}$ & $24 \cdot \overline{5}$ \\
\hline Cholesterol (mg) & $218 \cdot 1$ & $134 \cdot 7$ & $205 \cdot 3$ & $111 \cdot 0$ \\
\hline Dietary fibre (q: NDSR) & $19 \cdot 4$ & 8.9 & $19 \cdot 5$ & 8.9 \\
\hline Soluble fibre (g; NDSR) & $6 \cdot 7$ & $3 \cdot 1$ & $6 \cdot 7$ & $3 \cdot 0$ \\
\hline Insoluble fibre (g; NDSR) & $12 \cdot 6$ & $5 \cdot 9$ & $12 \cdot 7$ & $6 \cdot 0$ \\
\hline Carbohydrates (g) & $206 \cdot 0$ & $85 \cdot 0$ & $203 \cdot 8$ & $82 \cdot 2$ \\
\hline \multicolumn{5}{|l|}{ Micronutrients } \\
\hline Vitamin $A(\mu \mathrm{g} R E)$ & $1381 \cdot 4$ & $895 \cdot 2$ & $1390 \cdot 8$ & $946 \cdot 4$ \\
\hline$\beta$-Carotene $(\mu \mathrm{g})$ & $4364 \cdot 2$ & $3523 \cdot 4$ & $4419 \cdot 0$ & $3791 \cdot 1$ \\
\hline Vitamin C (mg) & $145 \cdot 2$ & $88 \cdot 3$ & $138 \cdot 7$ & 83.9 \\
\hline Vitamin D $(\mu \mathrm{g})$ & $4 \cdot 3$ & $2 \cdot 6$ & $4 \cdot 3$ & $2 \cdot 9$ \\
\hline Vitamin E (mg ATE) & $8 \cdot 3$ & $4 \cdot 4$ & $8 \cdot 1$ & $4 \cdot 2$ \\
\hline Thiamin (mg) & $1 \cdot 3$ & 0.5 & $1 \cdot 3$ & 0.5 \\
\hline Riboflavin (mg) & $1 \cdot 6$ & $0 \cdot 7$ & $1 \cdot 6$ & $0 \cdot 7$ \\
\hline Niacin $(m g)$ & $17 \cdot 8$ & $7 \cdot 0$ & $17 \cdot 6$ & $7 \cdot 1$ \\
\hline Vitamin $\mathrm{B}_{6}(\mathrm{mg})$ & $1 \cdot 7$ & $0 \cdot 7$ & $1 \cdot 7$ & $0 \cdot 7$ \\
\hline Vitamin $B_{12}(\mu \mathrm{g})$ & $4 \cdot 3$ & $2 \cdot 8$ & $4 \cdot 1$ & $2 \cdot 8$ \\
\hline $\mathrm{Ca}(\mathrm{mg})$ & $787 \cdot 7$ & $385 \cdot 1$ & $825 \cdot 6$ & $460 \cdot 2$ \\
\hline $\mathrm{Fe}(\mathrm{mg})$ & $11 \cdot 6$ & $4 \cdot 6$ & $11 \cdot 8$ & $4 \cdot 8$ \\
\hline $\mathrm{Na}(\mathrm{mg})$ & $2569 \cdot 4$ & $1094 \cdot 5$ & $2524 \cdot 6$ & $1060 \cdot 1$ \\
\hline Folate $(\mu \mathrm{g})$ & $316 \cdot 2$ & $144 \cdot 3$ & $311 \cdot 3$ & $140 \cdot 9$ \\
\hline Lycopene ( $\mu \mathrm{g} ;$ NDSR) & $6597 \cdot 3$ & $7425 \cdot 4$ & $5941 \cdot 7$ & $5222 \cdot 8$ \\
\hline Lutein/zeaxanthin ( $\mu \mathrm{g} ; \mathrm{NDSR}$ ) & $3017 \cdot 5$ & $3072 \cdot 2$ & $2928 \cdot 5$ & $3173 \cdot 7$ \\
\hline Methionine (g; NDSR) & $1 \cdot 4$ & 0.6 & $1 \cdot 4$ & 0.6 \\
\hline \multicolumn{5}{|l|}{ Selected supplement intakest } \\
\hline Vitamin A ( $\mu \mathrm{g} R E)$ & $955 \cdot 9$ & $563 \cdot 3$ & $1047 \cdot 2$ & $764 \cdot 5$ \\
\hline$\beta$-Carotene $(\mu \mathrm{g})$ & $191 \cdot 8$ & $199 \cdot 2$ & $217 \cdot 9$ & $259 \cdot 2$ \\
\hline Vitamin C (mg) & $251 \cdot 4$ & $327 \cdot 4$ & $245 \cdot 1$ & $351 \cdot 8$ \\
\hline Vitamin D $(\mu \mathrm{g})$ & $8 \cdot 7$ & $4 \cdot 5$ & $8 \cdot 8$ & $4 \cdot 7$ \\
\hline Vitamin E (mg ATE) & $43 \cdot 5$ & $60 \cdot 4$ & $54 \cdot 2$ & $66 \cdot 0$ \\
\hline Thiamin (mg) & $2 \cdot 5$ & $2 \cdot 5$ & $2 \cdot 7$ & $2 \cdot 6$ \\
\hline Riboflavin (mg) & $2 \cdot 2$ & $1 \cdot 7$ & $2 \cdot 3$ & $1 \cdot 7$ \\
\hline $\operatorname{Niacin}(\mathrm{mg})$ & $21 \cdot 1$ & $12 \cdot 2$ & $19 \cdot 9$ & $11 \cdot 0$ \\
\hline Vitamin $\mathrm{B}_{6}(\mathrm{mg})$ & $9 \cdot 2$ & $14 \cdot 9$ & $10 \cdot 0$ & $15 \cdot 5$ \\
\hline Vitamin $B_{12}(\mu \mathrm{g})$ & $4 \cdot 8$ & $2 \cdot 0$ & 4.9 & 1.9 \\
\hline $\mathrm{Ca}(\mathrm{mg})$ & $493 \cdot 3$ & $332 \cdot 2$ & $513 \cdot 7$ & $330 \cdot 7$ \\
\hline $\mathrm{Fe}(\mathrm{mg})$ & $17 \cdot 3$ & $8 \cdot 7$ & $16 \cdot 3$ & $7 \cdot 6$ \\
\hline Folate $(\mu \mathrm{g})$ & $327 \cdot 3$ & $142 \cdot 3$ & $327 \cdot 6$ & $137 \cdot 8$ \\
\hline
\end{tabular}

DHQ, diet history questionnaire; NDSR, Nutrition Data Systems for Research; RE, retinol equivalents; ATE, $\alpha$-tocopherol equivalents. ${ }^{\star}$ Cases: $1608 \cdot 6 \mathrm{~kJ}(627 \cdot 8 \mathrm{kcal})$; controls: $1597 \cdot 1 \mathrm{~kJ}(626 \cdot 3 \mathrm{kcal})$.

tEstimated among users of given supplement; multivitamin or nutrient-only-type source.

age, who were married or had been married previously (96\%; Table 1). Risk increased with being overweight or obese (BMI $\geq 25 \cdot 0 \mathrm{~kg} / \mathrm{m}^{2} v$. BMI $<25 \cdot 0 \mathrm{~kg} / \mathrm{m}^{2}$ ), having a waist circumference $\geq 88 \mathrm{~cm}$, having a younger age at menarche and having a history of hypertension or type 2 diabetes. Risk was reduced for women who were more educated, parous, past-year dietary supplement users, ever hormone contraception users or post- or perimenopausal and users of E + P HT (Table 1). Women were generally physically inactive and low alcohol consumers; half of them were never smokers (Table 1). The daily nutrient intakes reported by cases and controls are presented in Table 2 . The prevalence of use of any multi- vitamin or any nutrient-only-type supplement was $84 \%$ for cases and $88 \%$ for controls. Vitamin C was the most prevalent supplemental nutrient, taken by $69 \%$ of cases and $74 \%$ of controls.

\section{Macronutrients}

We did not find an association with EC for the majority of macronutrients examined, including total energy intake, protein, total fat, saturated fat, carbohydrate and total dietary fibre (Table 3). We observed a 51-59\% elevation in risk in the highest quartile of intake of dietary cholesterol, with a statistically significant increasing trend in both ageadjusted and multivariable-adjusted models $(\mathrm{OR}=1.59$, 
Table 3 Age- and multivariable-adjusted $\mathrm{OR}$ and $95 \% \mathrm{Cl}$ for risk of endometrial cancer with daily intakes of individual macronutrients

\begin{tabular}{|c|c|c|c|c|c|c|c|}
\hline Nutrient exposure quartile & Cases ( $n$ 506) & Controls ( $n$ 981) & Age-adjusted OR & $95 \% \mathrm{Cl}$ & Multivariable $C$ & ORt & $95 \% \mathrm{Cl}$ \\
\hline \multicolumn{8}{|l|}{ Total energy $(\mathrm{kJ}) \ddagger$} \\
\hline$\leq 4880 \cdot 6$ & 122 & 245 & $1 \cdot 00$ & - & $1 \cdot 00$ & & - \\
\hline$>4880 \cdot 6$ to $\leq 6230 \cdot 0$ & 129 & 246 & 1.06 & $0 \cdot 78,1 \cdot 44$ & 1.06 & & $0.74,1.51$ \\
\hline$>6230 \cdot 0$ to $\leq 7875 \cdot 1$ & 124 & 244 & 1.04 & $0 \cdot 76,1 \cdot 41$ & $1 \cdot 04$ & & $0.68,1.59$ \\
\hline$>7875 \cdot 1$ to $\leq 19318 \cdot 4$ & 131 & 246 & 1.09 & $0.80,1.48$ & 1.08 & & $0.56,2.09$ \\
\hline$P$ for trend & & & & 0.87 & & 0.97 & \\
\hline \multicolumn{8}{|l|}{ Protein $(\mathrm{g})$} \\
\hline$\leq 53.0$ & 121 & 246 & $1 \cdot 00$ & - & $1 \cdot 00$ & & - \\
\hline$>53 \cdot 0$ to $\leq 61 \cdot 1$ & 144 & 244 & $1 \cdot 21$ & $0.90,1.63$ & $1 \cdot 31$ & & $0.95,1.80$ \\
\hline$>61 \cdot 1$ to $\leq 68 \cdot 8$ & 106 & 246 & $0 \cdot 88$ & $0 \cdot 64,1 \cdot 21$ & 0.90 & & $0.64,1 \cdot 26$ \\
\hline$>68 \cdot 8$ to $\leq 101 \cdot 2$ & 135 & 245 & $1 \cdot 13$ & $0.83,1.53$ & $1 \cdot 10$ & & $0.79,1.52$ \\
\hline \multirow{2}{*}{\multicolumn{8}{|c|}{ Total fat $(\mathrm{g})$}} \\
\hline & & & & & & & \\
\hline$\leq 45 \cdot 7$ & 123 & 245 & $1 \cdot 00$ & - & $1 \cdot 00$ & & - \\
\hline$>45 \cdot 7$ to $\leq 53.5$ & 114 & 245 & 0.93 & $0 \cdot 68,1 \cdot 27$ & $1 \cdot 01$ & & $0.72,1 \cdot 41$ \\
\hline$>53 \cdot 5$ to $\leq 61 \cdot 9$ & 131 & 246 & 1.07 & $0.79,1 \cdot 45$ & $1 \cdot 13$ & & $0.81,1.56$ \\
\hline$>61.9$ to $\leq 120 \cdot 4$ & 138 & 245 & $1 \cdot 13$ & $0.84,1.53$ & $1 \cdot 12$ & & $0.80,1.55$ \\
\hline$P$ for trend & & & & 0.30 & & 0.39 & \\
\hline \multicolumn{8}{|l|}{ Saturated fat (g) } \\
\hline$\leq 14 \cdot 3$ & 120 & 245 & $1 \cdot 00$ & - & $1 \cdot 00$ & & - \\
\hline$>14 \cdot 3$ to $\leq 17 \cdot 0$ & 120 & 245 & $1 \cdot 01$ & $0.74,1 \cdot 37$ & $1 \cdot 08$ & & $0.78,1.51$ \\
\hline$>17 \cdot 0$ to $\leq 20 \cdot 5$ & 140 & 245 & $1 \cdot 18$ & $0.87,1.59$ & $1 \cdot 21$ & & $0.87,1.67$ \\
\hline$>20 \cdot 5$ to $\leq 44 \cdot 3$ & 126 & 246 & $1 \cdot 06$ & $0 \cdot 78,1 \cdot 44$ & $1 \cdot 06$ & & $0.76,1.49$ \\
\hline$P$ for trend & & & & 0.51 & & 0.59 & \\
\hline \multicolumn{8}{|l|}{ Monounsaturated fat $(\mathrm{g})$} \\
\hline$\leq 16 \cdot 9$ & 124 & 246 & 1.00 & - & 1.00 & & - \\
\hline$>16 \cdot 9$ to $\leq 20 \cdot 0$ & 124 & 245 & $1 \cdot 01$ & $0.75,1.37$ & 1.06 & & $0.76,1.47$ \\
\hline$>20 \cdot 0$ to $\leq 23 \cdot 4$ & 125 & 245 & 1.02 & $0.75,1 \cdot 38$ & 1.01 & & $0.72,1.40$ \\
\hline$>23.4$ to $\leq 45.9$ & 133 & 245 & 1.09 & $0.80,1 \cdot 47$ & 1.07 & & $0.77,1.48$ \\
\hline$P$ for trend & & & & 0.60 & & 0.78 & \\
\hline \multicolumn{8}{|l|}{ Polyunsaturated fat (g) } \\
\hline$\leq 9 \cdot 7$ & 121 & 245 & $1 \cdot 00$ & - & 1.00 & & - \\
\hline$>9 \cdot 7$ to $\leq 11 \cdot 7$ & 130 & 246 & 1.06 & $0.78,1.44$ & 1.07 & & $0.77,1.49$ \\
\hline$>11 \cdot 7$ to $\leq 13.9$ & 121 & 244 & $1 \cdot 00$ & $0 \cdot 74,1 \cdot 36$ & 0.97 & & $0 \cdot 70,1 \cdot 35$ \\
\hline$>13 \cdot 9$ to $\leq 40 \cdot 1$ & 134 & 246 & $1 \cdot 10$ & $0.81,1 \cdot 49$ & $1 \cdot 11$ & & $0.81,1.54$ \\
\hline$P$ for trend & & & & 0.64 & & 0.66 & \\
\hline \multicolumn{8}{|l|}{ Discretionary fat (g) } \\
\hline$\leq 34 \cdot 3$ & 118 & 246 & $1 \cdot 00$ & - & $1 \cdot 00$ & & - \\
\hline$>34 \cdot 3$ to $\leq 41 \cdot 3$ & 117 & 245 & $1 \cdot 01$ & $0 \cdot 74,1 \cdot 37$ & $1 \cdot 06$ & & $0 \cdot 76,1 \cdot 48$ \\
\hline$>41 \cdot 3$ to $\leq 49 \cdot 6$ & 141 & 244 & $1 \cdot 21$ & $0 \cdot 89,1 \cdot 64$ & $1 \cdot 23$ & & $0 \cdot 89,1 \cdot 71$ \\
\hline$>49 \cdot 6$ to $\leq 101 \cdot 1$ & 130 & 246 & $1 \cdot 11$ & $0 \cdot 82,1 \cdot 51$ & $1 \cdot 12$ & & $0.80,1.56$ \\
\hline$P$ for trend & & & & $0 \cdot 31$ & & 0.36 & \\
\hline \multicolumn{8}{|l|}{ Cholesterol (mg) } \\
\hline$\leq 141 \cdot 2$ & 100 & 246 & 1.00 & - & 1.00 & & - \\
\hline$>141 \cdot 2$ to $\leq 179 \cdot 3$ & 126 & 245 & $1 \cdot 27$ & $0.92,1 \cdot 74$ & $1 \cdot 22$ & & $0.87,1 \cdot 72$ \\
\hline$>179 \cdot 3$ to $\leq 225 \cdot 4$ & 123 & 245 & $1 \cdot 24$ & $0.90,1.71$ & 1.29 & & $0.92,1.82$ \\
\hline$>225 \cdot 4$ to $\leq 917 \cdot 8$ & 157 & 245 & 1.59 & $1 \cdot 17,2 \cdot 16$ & $1 \cdot 51$ & & $1 \cdot 08,2 \cdot 11$ \\
\hline$P$ for trend & & & & 0.01 & & 0.02 & \\
\hline \multicolumn{8}{|l|}{ Carbohydrate (g) } \\
\hline$\leq 171 \cdot 7$ & 127 & 245 & $1 \cdot 00$ & - & $1 \cdot 00$ & & - \\
\hline$>171 \cdot 7$ to $\leq 195 \cdot 8$ & 128 & 245 & $1 \cdot 01$ & $0.75,1.37$ & $1 \cdot 10$ & & $0.79,1.51$ \\
\hline$>195 \cdot 8$ to $\leq 215 \cdot 8$ & 113 & 245 & 0.88 & $0 \cdot 65,1 \cdot 21$ & 0.97 & & $0.70,1.35$ \\
\hline$>215 \cdot 8$ to $\leq 332 \cdot 0$ & 138 & 246 & 1.07 & $0.79,1.45$ & $1 \cdot 11$ & & $0.80,1.54$ \\
\hline$P$ for trend & & & & 0.86 & & 0.70 & \\
\hline Dietary fibre (g; NDSR) & & & & & & & \\
\hline$\leq 14 \cdot 8$ & 132 & 246 & $1 \cdot 00$ & - & $1 \cdot 00$ & & - \\
\hline$>14 \cdot 8$ to $\leq 18 \cdot 4$ & 134 & 245 & 1.01 & $0.75,1 \cdot 36$ & $1 \cdot 13$ & & $0.82,1.56$ \\
\hline$>18.4$ to $\leq 21.9$ & 120 & 244 & 0.90 & $0.66,1 \cdot 22$ & 1.06 & & $0.77,1.48$ \\
\hline$>21.9$ to $\leq 45 \cdot 1$ & 120 & 246 & 0.88 & $0.65,1.20$ & 0.96 & & $0.69,1.34$ \\
\hline$P$ for trend & & & & 0.32 & & 0.75 & \\
\hline Insoluble fibre (g; NDSR) & & & & & & & \\
\hline$\leq 9 \cdot 5$ & 132 & 246 & $1 \cdot 00$ & - & 1.00 & & - \\
\hline$>9 \cdot 5$ to $\leq 11 \cdot 8$ & 129 & 245 & 0.97 & $0 \cdot 72,1 \cdot 31$ & $1 \cdot 16$ & & $0.84,1.60$ \\
\hline$>11 \cdot 8$ to $\leq 14.3$ & 132 & 245 & 0.98 & $0.73,1 \cdot 33$ & $1 \cdot 14$ & & $0.82,1.58$ \\
\hline$>14 \cdot 3$ to $\leq 31 \cdot 5$ & 113 & 245 & 0.84 & $0 \cdot 61,1 \cdot 14$ & 0.95 & & $0.68,1.34$ \\
\hline$P$ for trend & & & & 0.30 & & 0.81 & \\
\hline Soluble fibre (g; NDSR) & & & & & & & \\
\hline$\leq 5 \cdot 1$ & 116 & 246 & $1 \cdot 00$ & - & $1 \cdot 00$ & & - \\
\hline$>5 \cdot 1$ to $\leq 6 \cdot 3$ & 148 & 244 & $1 \cdot 27$ & $0.94,1 \cdot 72$ & $1 \cdot 40$ & & $1.01,1.93$ \\
\hline$>6.3$ to $\leq 7.5$ & 124 & 246 & 1.05 & $0.77,1.43$ & $1 \cdot 20$ & & $0.86,1.68$ \\
\hline$>7.5$ to $\leq 14.9$ & 118 & 245 & $1 \cdot 00$ & $0.73,1 \cdot 36$ & $1 \cdot 08$ & & $0.77,1.52$ \\
\hline$P$ for trend & & & & 0.65 & & 0.91 & \\
\hline
\end{tabular}

NDSR, Nutrition Data Systems for Research.

${ }^{*}$ Adjusted for age (years) and total energy intake (kcal/d; except in the case of total energy intake).

†Additionally adjusted for age at menarche (years), BMl $\left(<25 \cdot 0 \mathrm{v} .25 \cdot 0-29 \cdot 9, \geq 30 \cdot 0 \mathrm{~kg} / \mathrm{m}^{2}\right)$, parity $(0 \mathrm{v} .1-2,>2$ pregnancies at $\geq 20$ weeks' gestation), educational level (below high school $v$. high school or above), hypertension history (ever $v$. never), hormone contraceptive use (never $v$. ever), hormone therapy use combined with menopausal status (post/perimenopausal/no hormone therapy $v$. post/perimenopausal + oestrogen, post/perimenopausal + oestrogen + progesterone, post/perimenopausal + other menopausal hormones and premenopausal), alcohol consumption (0 $\mathrm{v}$. $<1 \mathrm{drink}$, $\geq 1 \mathrm{drink} / \mathrm{d})$. $\ddagger$ Quartile cut-off points in kcal: $1166 \cdot 5,1489 \cdot 0,1882 \cdot 2$ and $4617 \cdot 2$, respectively. 
Table 4 Multivariable-adjusted OR and $95 \% \mathrm{Cl}$ for risk of endometrial cancer with cholesterol intake (mg) stratified by menopausal status $(n 1485)$, BMI ( $n$ 1486) and HT use $(n 876)$

\begin{tabular}{|c|c|c|c|c|c|c|c|c|}
\hline & \multicolumn{4}{|c|}{ Pre/perimenopausal ( $n$ 168) } & \multicolumn{4}{|c|}{ Postmenopausal ( $n$ 1317) } \\
\hline & Cases (n 52) & Controls ( $n$ 116) & Multivariable-adjusted $\mathrm{OR}^{*}$ & $95 \% \mathrm{Cl}$ & Cases ( $n$ 453) & Controls ( $n$ 864) & Multivariable-adjusted $\mathrm{OR}^{\star}+$ & $95 \% \mathrm{Cl}$ \\
\hline \multicolumn{9}{|l|}{ Cholesterol (mg) } \\
\hline$\leq 141 \cdot 2$ & 21 & 30 & $1 \cdot 00$ & - & 79 & 186 & $1 \cdot 00$ & - \\
\hline$>141 \cdot 2$ to $\leq 179 \cdot 3$ & 31 & 59 & $1 \cdot 61$ & $0.73,3.54$ & 94 & 186 & $1 \cdot 16$ & $0 \cdot 79,1 \cdot 70$ \\
\hline$>179 \cdot 3$ to $\leq 225 \cdot 4$ & 35 & 75 & $2 \cdot 10$ & $0.98,4.51$ & 88 & 170 & $1 \cdot 14$ & $0 \cdot 77,1 \cdot 68$ \\
\hline$>225 \cdot 4$ to $\leq 917 \cdot 8$ & 32 & 72 & $1 \cdot 55$ & $0.72,3.35$ & 125 & 172 & 1.51 & $1 \cdot 04,2 \cdot 20$ \\
\hline$P$ for trend & \multicolumn{4}{|c|}{$0 \cdot 24$} & \multicolumn{4}{|c|}{0.04} \\
\hline & \multicolumn{4}{|c|}{$\mathrm{BMI}<25 \cdot 0 \mathrm{~kg} / \mathrm{m}^{2}(n 413)$} & \multicolumn{4}{|c|}{$\mathrm{BMI} \geq 25 \cdot 0 \mathrm{~kg} / \mathrm{m}^{2}(n$ 1073) } \\
\hline & Cases (n 92) & Controls ( $n$ 321) & Multivariable-adjusted $\mathrm{OR}^{*}$ & $95 \% \mathrm{Cl}$ & Cases ( $n$ 414) & Controls $(n$ 659) & Multivariable-adjusted $\mathrm{OR}^{\star}$ & $95 \% \mathrm{Cl}$ \\
\hline \multicolumn{9}{|l|}{ Cholesterol (mg) } \\
\hline$\leq 141 \cdot 2$ & 29 & 93 & $1 \cdot 00$ & - & 71 & 153 & $1 \cdot 00$ & - \\
\hline$>141 \cdot 2$ to $\leq 179 \cdot 3$ & 30 & 68 & $1 \cdot 34$ & $0 \cdot 70,2 \cdot 58$ & 96 & 177 & $1 \cdot 29$ & $0.87,1.92$ \\
\hline$>179 \cdot 3$ to $\leq 225 \cdot 4$ & 18 & 86 & 0.67 & $0 \cdot 33,1 \cdot 36$ & 105 & 159 & $1 \cdot 69$ & $1 \cdot 13,2 \cdot 52$ \\
\hline$>225 \cdot 4$ to $\leq 917 \cdot 8$ & 15 & 74 & $0 \cdot 65$ & $0 \cdot 31,1 \cdot 35$ & 142 & 170 & $2 \cdot 11$ & $1 \cdot 44,3 \cdot 10$ \\
\hline \multirow[t]{3}{*}{$P$ for trend } & \multicolumn{4}{|c|}{$0 \cdot 11$} & \multicolumn{4}{|c|}{0.00} \\
\hline & \multicolumn{4}{|c|}{ No HT ( $n$ 581) } & \multicolumn{4}{|c|}{$\mathrm{E}+\mathrm{P} \mathrm{HT}(n$ 295) } \\
\hline & Cases $(n 226)$ & Controls ( $n$ 355) & Multivariable-adjusted $\mathrm{OR}^{*}$ & $95 \% \mathrm{Cl}$ & Cases $(n 76)$ & Controls ( $n$ 219) & Multivariable-adjusted $\mathrm{OR}^{\star}$ & $95 \% \mathrm{Cl}$ \\
\hline \multicolumn{9}{|l|}{ Cholesterol (mg) } \\
\hline$\leq 141 \cdot 2$ & 44 & 91 & $1 \cdot 00$ & - & 20 & 55 & 1.00 & - \\
\hline$>141 \cdot 2$ to $\leq 179 \cdot 3$ & 51 & 99 & 1.03 & $0.60,1 \cdot 75$ & 21 & 54 & $1 \cdot 17$ & $0.55,2 \cdot 47$ \\
\hline$>179 \cdot 3$ to $\leq 225 \cdot 4$ & 45 & 82 & $1 \cdot 08$ & $0 \cdot 62,1 \cdot 90$ & 15 & 59 & $0 \cdot 68$ & $0.31,1.50$ \\
\hline$>225 \cdot 4$ to $\leq 917 \cdot 8$ & 86 & 83 & $1 \cdot 79$ & $1 \cdot 06,3 \cdot 00$ & 20 & 51 & $1 \cdot 08$ & $0.50,2 \cdot 31$ \\
\hline$P$ for trend & \multicolumn{4}{|c|}{0.02} & & & \multicolumn{2}{|l|}{$0 \cdot 80$} \\
\hline
\end{tabular}

$\mathrm{HT}$, hormone therapy; $\mathrm{E}+\mathrm{P}$, oestrogen + progesterone.

${ }^{*}$ Adjusted for age (years) and total energy intake (kJ (kcal)), age at menarche (years), BMI ( $\left.<25 \cdot 0 \mathrm{v} \cdot 25 \cdot 0-29 \cdot 9, \geq 30 \cdot 0 \mathrm{~kg} / \mathrm{m}^{2}\right)$, parity $(0 \mathrm{v} .1-2,>2$ pregnancies at $\geq 20$ weeks' gestation), educational level (below high school $v$. high school or above), hypertension history (ever $v$. never), hormone contraceptive use (never $v$. ever), alcohol consumption $(0$ drink $v$. $<1$ drink, $\geq 1$ drink/d).

tlf further adjusted for use of HT (lowest to highest quartile of cholesterol intake): OR=1.14 (95\% Cl 0.78, 1.67), $1 \cdot 16(95 \% \mathrm{Cl} 0.78,1 \cdot 72)$ and $1 \cdot 48(95 \% \mathrm{Cl} 1 \cdot 02,2 \cdot 16)$. 


\begin{tabular}{|c|c|c|c|c|c|c|c|c|c|c|c|}
\hline \multicolumn{6}{|c|}{ Diet alone $^{*}$} & \multicolumn{6}{|c|}{ Diet with supplementst } \\
\hline Nutrient exposure quartile & $\begin{array}{l}\text { Cases } \\
(n 506)\end{array}$ & $\begin{array}{l}\text { Controls } \\
(n \text { 981) }\end{array}$ & $\begin{array}{c}\text { Multivariable } \\
\text { OR }\end{array}$ & & $95 \% \mathrm{Cl}$ & Nutrient exposure quartile & $\begin{array}{l}\text { Cases } \\
(n 506)\end{array}$ & $\begin{array}{l}\text { Controls } \\
(n 981)\end{array}$ & $\begin{array}{c}\text { Multivariable } \\
\text { OR }\end{array}$ & & $95 \% \mathrm{Cl}$ \\
\hline \multicolumn{12}{|l|}{ Vitamin A (RE) } \\
\hline$\leq 838.6$ & 120 & 246 & $1 \cdot 00$ & & - & $\leq 1115 \cdot 5$ & 136 & 245 & $1 \cdot 00$ & & - \\
\hline$>838 \cdot 6$ to $\leq 1115 \cdot 1$ & 119 & 244 & 0.97 & & $0 \cdot 70,1 \cdot 36$ & $>1115 \cdot 5$ to $\leq 1800 \cdot 1$ & 127 & 246 & 0.98 & & $0 \cdot 71,1 \cdot 34$ \\
\hline$>1115 \cdot 1$ to $\leq 1603 \cdot 0$ & 150 & 246 & $1 \cdot 30$ & & $0.94,1.79$ & $>1800 \cdot 1$ to $\leq 2380 \cdot 2$ & 127 & 245 & $1 \cdot 11$ & & $0.80,1.53$ \\
\hline$>1603 \cdot 0$ to $\leq 8028 \cdot 0$ & 117 & 245 & $1 \cdot 03$ & & $0 \cdot 74,1 \cdot 45$ & $>2380 \cdot 2$ to $\leq 9135 \cdot 3$ & 116 & 245 & 0.95 & & $0 \cdot 68,1 \cdot 32$ \\
\hline$P$ for trend & & & & 0.44 & & $P$ for trend & & & & 0.97 & \\
\hline \multicolumn{12}{|l|}{$\beta$-Carotene $(\mu \mathrm{g})$} \\
\hline$\leq 2122 \cdot 7$ & 121 & 246 & $1 \cdot 00$ & & - & $\leq 2258 \cdot 0$ & 112 & 246 & $1 \cdot 00$ & & - \\
\hline$>2122 \cdot 7$ to $\leq 3302 \cdot 5$ & 117 & 244 & $1 \cdot 01$ & & $0 \cdot 73,1 \cdot 41$ & $>2258 \cdot 0$ to $\leq 3438 \cdot 4$ & 117 & 244 & 0.95 & & $0 \cdot 68,1 \cdot 32$ \\
\hline$>3302.5$ to $\leq 5199.5$ & 147 & 245 & $1 \cdot 22$ & & $0.89,1.69$ & $>3438 \cdot 4$ to $\leq 5371 \cdot 0$ & 147 & 246 & $1 \cdot 21$ & & $0.88,1.67$ \\
\hline$>5199.5$ to $\leq 28673.0$ & 121 & 246 & $1 \cdot 14$ & & $0.81,1.59$ & $>5371.0$ to $\leq 28673.1$ & 120 & 245 & $1 \cdot 10$ & & $0.79,1.53$ \\
\hline$P$ for trend & & & & $0 \cdot 27$ & & $P$ for trend & & & & $0 \cdot 32$ & \\
\hline \multicolumn{12}{|l|}{ Vitamin C (mg) } \\
\hline$\leq 87.6$ & 108 & 246 & $1 \cdot 00$ & & - & $\leq 131 \cdot 8$ & 136 & 246 & 1.00 & & - \\
\hline$>87 \cdot 6$ to $\leq 124 \cdot 4$ & 131 & 244 & $1 \cdot 25$ & & $0.90,1 \cdot 75$ & $>131 \cdot 8$ to $\leq 185 \cdot 5$ & 107 & 244 & 0.90 & & $0 \cdot 64,1 \cdot 25$ \\
\hline$>124 \cdot 4$ to $\leq 167 \cdot 0$ & 129 & 246 & $1 \cdot 20$ & & $0.86,1.68$ & $>185 \cdot 5$ to $\leq 374.7$ & 153 & 246 & $1 \cdot 28$ & & $0.94,1.76$ \\
\hline$>167.0$ to $\leq 478.2$ & 138 & 245 & 1.45 & & $1.04,2 \cdot 03$ & $>374 \cdot 7$ to $\leq 2465 \cdot 7$ & 110 & 245 & 0.84 & & $0.60,1.17$ \\
\hline$P$ for trend & & & & 0.05 & & $P$ for trend & & & & $0 \cdot 84$ & \\
\hline \multicolumn{12}{|l|}{ Vitamin D $(\mu \mathrm{g})$} \\
\hline$\leq 2 \cdot 4$ & 126 & 245 & $1 \cdot 00$ & & - & $\leq 4 \cdot 4$ & 133 & 246 & $1 \cdot 00$ & & - \\
\hline$>2 \cdot 4$ to $\leq 3.4$ & 117 & 246 & 0.88 & & $0.63,1.22$ & $>4.4$ to $\leq 10 \cdot 2$ & 132 & 245 & 1.07 & & $0.77,1.47$ \\
\hline$>3 \cdot 4$ to $\leq 5 \cdot 1$ & 139 & 245 & $1 \cdot 10$ & & $0.80,1.52$ & $>10 \cdot 2$ to $\leq 14 \cdot 0$ & 116 & 244 & 0.97 & & $0 \cdot 70,1 \cdot 34$ \\
\hline$>5 \cdot 1$ to $\leq 18 \cdot 1$ & 124 & 245 & 0.98 & & $0.71,1 \cdot 36$ & $>14 \cdot 0$ to $\leq 33 \cdot 1$ & 125 & 246 & 1.08 & & $0.78,1.50$ \\
\hline$P$ for trend & & & & 0.74 & & $P$ for trend & & & & $0 \cdot 78$ & \\
\hline \multicolumn{12}{|l|}{ Vitamin E (mg ATE) } \\
\hline$\leq 6 \cdot 2$ & 127 & 245 & $1 \cdot 00$ & & - & $\leq 7 \cdot 9$ & 133 & 246 & $1 \cdot 00$ & & - \\
\hline$>6 \cdot 2$ to $\leq 7 \cdot 3$ & 128 & 246 & 1.08 & & $0.78,1.49$ & $>7 \cdot 9$ to $\leq 15 \cdot 2$ & 147 & 244 & $1 \cdot 17$ & & $0.85,1.60$ \\
\hline$>7 \cdot 3$ to $\leq 8 \cdot 5$ & 115 & 245 & $1 \cdot 01$ & & $0 \cdot 73,1 \cdot 41$ & $>15 \cdot 2$ to $\leq 66 \cdot 6$ & 136 & 245 & $1 \cdot 13$ & & $0 \cdot 82,1 \cdot 55$ \\
\hline$>8 \cdot 5$ to $\leq 18 \cdot 7$ & 136 & 245 & $1 \cdot 18$ & & $0.85,1.62$ & $>66 \cdot 6$ to $\leq 324.4$ & 90 & 246 & 0.74 & & $0.52,1.04$ \\
\hline$P$ for trend & & & & 0.41 & & $P$ for trend & & & & $0 \cdot 13$ & \\
\hline \multicolumn{12}{|l|}{ Folate ( $\mu \mathrm{g}$ DFE) } \\
\hline$\leq 277.6$ & 133 & 246 & $1 \cdot 00$ & & - & $\leq 329 \cdot 9$ & 125 & 245 & $1 \cdot 00$ & & - \\
\hline$>277.6$ to $\leq 322.5$ & 97 & 245 & 0.80 & & $0.57,1.12$ & $>329.9$ to $\leq 591.8$ & 139 & 246 & $1 \cdot 14$ & & $0.82,1.57$ \\
\hline$>322.5$ to $\leq 377.6$ & 140 & 245 & $1 \cdot 14$ & & $0.83,1.57$ & $>591 \cdot 8$ to $\leq 987 \cdot 7$ & 111 & 244 & 0.95 & & $0 \cdot 68,1.32$ \\
\hline$>377 \cdot 6$ to $\leq 851 \cdot 3$ & 136 & 245 & $1 \cdot 18$ & & $0.85,1.63$ & $>987 \cdot 7$ to $\leq 1551 \cdot 0$ & 131 & 246 & $1 \cdot 18$ & & $0.85,1.64$ \\
\hline$P$ for trend & & & & 0.12 & & $P$ for trend & & & & 0.54 & \\
\hline \multicolumn{12}{|l|}{ Thiamin (mg) } \\
\hline$\leq 1 \cdot 1$ & 119 & 246 & $1 \cdot 00$ & & - & $\leq 1 \cdot 3$ & 139 & 245 & $1 \cdot 00$ & & - \\
\hline$>1 \cdot 1$ to $\leq 1 \cdot 2$ & 108 & 244 & 0.93 & & $0 \cdot 66,1 \cdot 30$ & $>1 \cdot 3$ to $\leq 2 \cdot 2$ & 130 & 246 & 0.85 & & $0.62,1 \cdot 17$ \\
\hline$>1 \cdot 2$ to $\leq 1 \cdot 4$ & 161 & 245 & $1 \cdot 49$ & & $1 \cdot 09,2 \cdot 04$ & $>2.2$ to $\leq 2.9$ & 123 & 244 & 0.92 & & $0.67,1.27$ \\
\hline$>1.4$ to $\leq 2.5$ & 118 & 246 & $1 \cdot 01$ & & $0.72,1.41$ & $>2.9$ to $\leq 9.7$ & 114 & 246 & 0.83 & & $0 \cdot 60,1 \cdot 16$ \\
\hline$P$ for trend & & & & 0.30 & & $P$ for trend & & & & $0 \cdot 38$ & \\
\hline \multicolumn{12}{|l|}{ Riboflavin (mg) } \\
\hline$\leq 1 \cdot 3$ & 142 & 246 & $1 \cdot 00$ & & - & $\leq 1 \cdot 6$ & 138 & 246 & $1 \cdot 00$ & & - \\
\hline$>1 \cdot 3$ to $\leq 1 \cdot 5$ & 97 & 244 & 0.66 & & $0.47,0.92$ & $>1 \cdot 6$ to $\leq 2 \cdot 6$ & 133 & 245 & 1.07 & & $0.78,1.48$ \\
\hline$>1.5$ to $\leq 1.7$ & 136 & 246 & 1.00 & & $0.73,1.37$ & $>2.6$ to $\leq 3.5$ & 130 & 245 & $1 \cdot 12$ & & $0.82,1.55$ \\
\hline
\end{tabular}




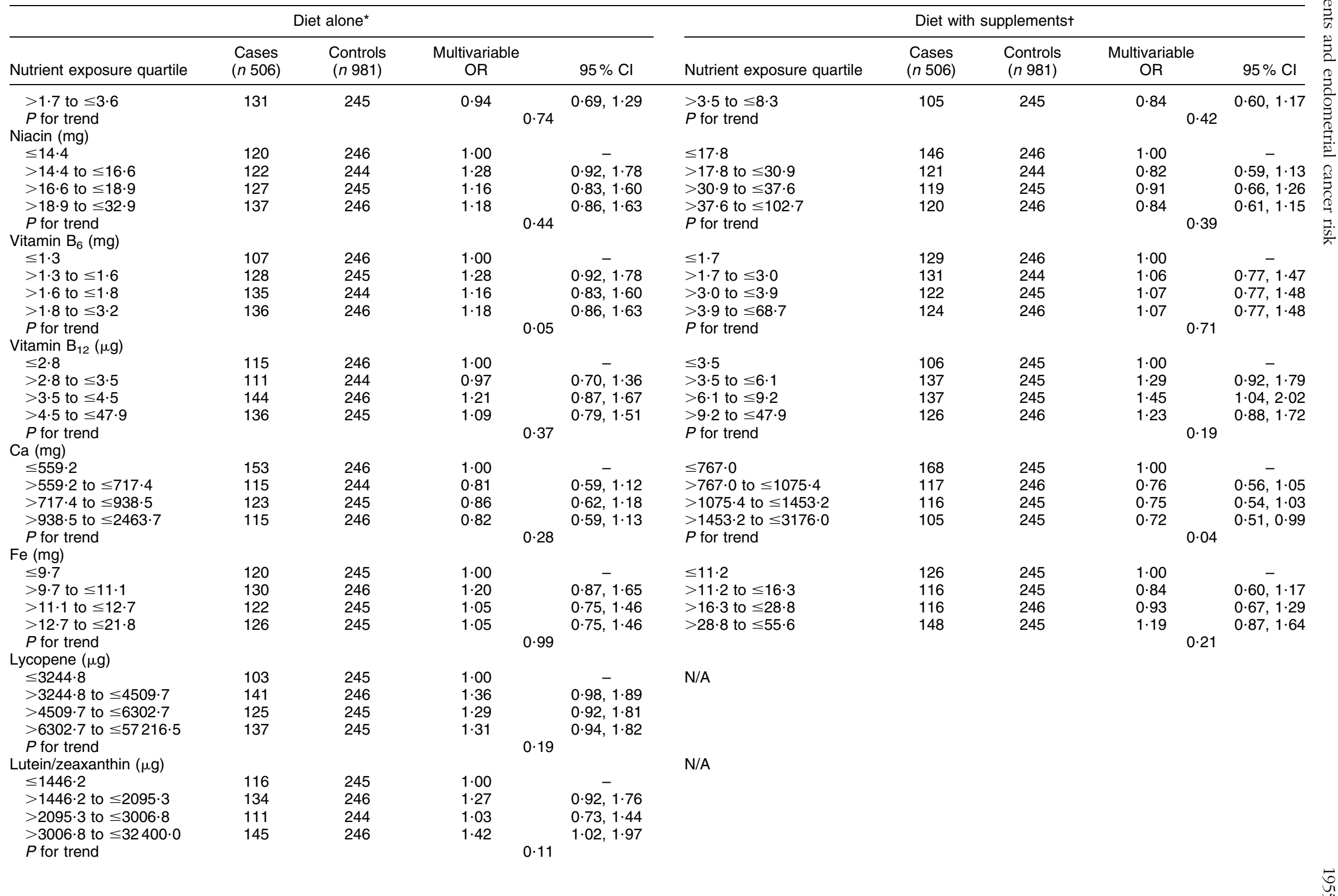


$95 \%$ CI $1 \cdot 17,2 \cdot 16, P$ for trend $=0 \cdot 01 ;$ OR $=1 \cdot 51,95 \%$ CI $1 \cdot 08,2 \cdot 11, P$ for trend $=0 \cdot 02$, respectively). BMI modified the association at the highest levels of cholesterol intake and EC ( $P$ for interaction $=0.01$ and $0 \cdot 00)$. We observed an 80-211\% statistically significant elevation in risk at the highest level of cholesterol intake for women whose BMI was defined as overweight or obese (BMI $\geq 25.0 \mathrm{~kg} / \mathrm{m}^{2}$; $\mathrm{OR}=2 \cdot 11,95 \%$ CI $1 \cdot 44,3 \cdot 10$ ) but not for normal-weight women (BMI $<25 \cdot 0 \mathrm{~kg} / \mathrm{m}^{2}$; Table 4). A similar pattern of significantly increased risk was observed with waist circumference $\geq 88 \mathrm{~cm}(\mathrm{OR}=2 \cdot 07,95 \%$ CI $1 \cdot 32,3 \cdot 25$; results not shown). We found no statistical interaction between cholesterol intake and menopausal status. However, when models were stratified, risks remained statistically significantly elevated among postmenopausal women in the highest quartile of cholesterol intake (OR $=1 \cdot 51,95 \% \mathrm{CI}$ $1 \cdot 04,2 \cdot 20, P$ for trend $=0 \cdot 04$ ). The association with cholesterol was also strengthened among postmenopausal women who were non-users of HT (OR $=1 \cdot 79,95 \%$ CI 1·06, 3.00, $P$ for trend $=0 \cdot 02)$ at the highest level of intake when it was examined in models assessing no HT and $\mathrm{E}+\mathrm{P}$ HT use.

\section{Micronutrients}

We did not observe an association with EC for most micronutrients (Table 5). A reduced risk was detected for dietary intake of riboflavin (multivariable-adjusted $\mathrm{OR}=$ $0 \cdot 66,95 \% \mathrm{CI} 0 \cdot 47,0 \cdot 92$ ) in the second, compared with the lowest, quartile of intake, but risk was unchanged for the remaining intake levels and there was no evidence for linear trend. Risk was reduced in the highest quartile of vitamin $\mathrm{E}$ intake from food and supplements in the age-adjusted model (OR $=0 \cdot 66,95 \%$ CI $0 \cdot 48,0 \cdot 91$, $P$ for trend $=0 \cdot 01$ ) but was attenuated in the multivariableadjusted model $(\mathrm{OR}=0.74,95 \% \mathrm{CI} 0.52,1.04, P$ for trend $=0 \cdot 13$ ). Age-adjusted risk accounting for supplement use at the highest level of intake was significantly reduced for $\mathrm{Ca}$ from food sources (OR $=0.73,95 \% \mathrm{CI}$ $0.54,0.99, P$ for trend $=0.07)$ but was attenuated in the multivariable-adjusted model (OR $=0.82,95 \%$ CI 0.59 , $1 \cdot 13, \quad P$ for trend $=0 \cdot 28$ ). The strongest association observed was for total combined $\mathrm{Ca}$ intake (from both food and supplements), in which we observed a statistically significant $28-40 \%$ reduced risk associated with the highest exposure level (multivariable-adjusted $\mathrm{OR}=0 \cdot 72$, $95 \%$ CI $0.51,0.99, P$ for trend $=0.04)$. In an analysis of $\mathrm{Ca}$ intake from food, restricted to supplement non-users ( $n 196$ cases, $n 300$ controls), using quartile cut-off points among non-supplement-using controls we found a statistically significant reduced risk for $\mathrm{EC}(\mathrm{OR}=0.52$, $95 \%$ CI, $0 \cdot 30,0.93, P$ for trend $=0 \cdot 07$ ). We observed unexpected increased risks at limited levels of intake of dietary soluble fibre, vitamin $\mathrm{C}$, thiamin, vitamin $\mathrm{B}_{6}$ and lutein/zeaxanthin and vitamin $\mathrm{B}_{12}$ from food plus supplements (Table 5). There was no evidence for linear trend being associated with these increased risks, except for vitamin $\mathrm{B}_{6}(P$ for trend $=0 \cdot 05)$. 


\section{Discussion}

In this population-based case-control study that had detailed assessments of food and supplement use, we did not find increased risks associated with most macronutrients, including total fat and saturated fat, but did find an association between decreased risks and some micronutrient intakes. Our findings are not corroborated by previous research, which has generally suggested a relationship between increased EC risks and higher intakes of total fat ${ }^{(20-26)}$, saturated fat ${ }^{(5,20,22,27)}$ and animal fat ${ }^{(5,20,25,26,28)}$. A meta-analysis reported statistically significant increased risks of $24-72 \%$ for total fat, $28-49 \%$ for saturated fat and $34-78 \%$ for animal fat ${ }^{(29)}$. Two studies have since found an association between non-statistically significant increased risk and higher total fat intake ${ }^{(30,31)}$ and one found no association $^{(32)}$. Meanwhile, our results are consistent with the Women's Health Initiative Dietary Intervention Trial, a study that aimed to reduce fat intake to $<20 \%$ of total energy intake from a baseline of $\geq 32 \%$ and found no change in EC incidence among postmenopausal women ${ }^{(33)}$.

The most pronounced finding among the macronutrients was a statistically significant increased risk of 51-59\% at the highest level of cholesterol intake. The association between dietary cholesterol and EC has been assessed in nine previous case-control studies ${ }^{(20,22-25,28,31,32,34)}$ with mixed results: three found statistically significant increased risks $^{(20,24,32)}$, but the remaining six found non-significant reduced risks ${ }^{(23,25)}$, no association ${ }^{(34)}$ or non-significant increased risks ${ }^{(22,28,31)}$. A pooled analysis of six of these studies reported a non-statistically significant increased risk of $35-39 \%$ with higher cholesterol intake ${ }^{(29)}$. Studies of serum cholesterol have shown similar mixed results ${ }^{(35-41)}$.

Our results are consistent with the increased EC risk observed for dietary cholesterol from pooled estimates. Although most cholesterol is produced by the liver, prolonged intake of dietary saturated fat and cholesterol raises the average serum cholesterol concentration, as well as levels of LDL cholesterol and HDL cholesterol ${ }^{(42)}$. Individual serum cholesterol response to intake of dietary cholesterol seems to be dependent on genetic susceptibility $^{(42)}$, which may, in part, contribute to mixed results from observational studies. The role of cholesterol in endometrial carcinogenesis is biologically plausible, as cholesterol and oestrogen are physiologically interconnected. Cholesterol is the founding substrate in steroid hormone synthesis $^{(43)}$ and can be converted to oestrogen via metabolic pathways; thus, increased levels of cholesterol may influence EC risk by increasing bioavailable oestrogen synthesis. Meanwhile, in postmenopausal women, circulating cholesterol levels are decreased as a result of oestrogen therapy ${ }^{(44)}$. Further, oestrogen stimulates LDL receptor activity; hence, premenopausal women have lower LDL cholesterol levels, which increase after menopause ${ }^{(45)}$. During premenopause, oestrogen production occurs in the ovaries and circulating oestrogen levels are tightly regulated; hence, any influence of cholesterol on oestrogen levels may be marginal ${ }^{(46)}$. Since endogenous oestrogen production after menopause occurs primarily in adipose tissue ${ }^{(46)}$, the relative influence of cholesterol on oestrogen bioavailability is greater than that before menopause. In addition, the oestrogen in HT users may overwhelm any effect of cholesterol. Our finding of a stronger increased risk with dietary cholesterol among postmenopausal women, particularly those not exposed to HT, and among overweight and obese women, is consistent with this hypothesis and suggests that cholesterol may be more influential after menopause. Alternatively, the highest sources of cholesterol among food groups in our questionnaire were, as expected, red meat (including higher fat beef, veal and pork), eggs, poultry and high-fat dairy. Hence, the increased risk with cholesterol may also reflect intake of animal foods that also increase EC risk ${ }^{(47)}$.

Numerous observational studies have reported on the influence of dietary micronutrients in EC aetiology $^{(5,6,20,21,23-26,28,30,31,34,48-53)}$; two were cohort studies ${ }^{(6,48)}$ and the rest were case-control studies. Reduced risks have been observed for dietary intake of vitamin $C^{(20,21,23,30,51,52)}$, $\beta$-carotene $\mathrm{e}^{(20,23,30,31,51-53)}$ or other carotenoids ${ }^{(20,23,28,31,50,53)}$, as well as for intakes of vitamin $\mathrm{A}^{(20,21,30)}$, folate ${ }^{(23,31)}$, $\mathrm{Ca}^{(25,34)}$, vitamins $\mathrm{E}^{(5,31)}, \mathrm{D}$ and $\mathrm{B}_{12}{ }^{(54)}$ and lycopene ${ }^{(48)}$. Pooled analyses suggest a reduced risk with intakes of $\beta$-carotene (12\%) and vitamins C (15\%) and E (9\%) from food $^{(55)}$. A hospital-based case-control study in Korea examined pre-operative levels of plasma micronutrients and found significantly reduced risks associated with higher concentrations of plasma $\beta$-carotene and lycopene $(\mathrm{OR}=0 \cdot 12,95 \%$ CI $0 \cdot 03,0 \cdot 48$ and $\mathrm{OR}=0 \cdot 15,95 \% \mathrm{CI}$ $0 \cdot 04,0 \cdot 61$, respectively $)^{(56)}$. Overall, the evidence from dietary studies is mixed, which may be explained by misclassification of exposure levels or by confounding by unmeasured supplement use. The benefits of supplements on cancer risk are inconclusive; supplements are currently not recommended for cancer prevention ${ }^{(3)}$. A recent meta-analysis of randomized controlled trials showed no benefit of antioxidant supplementation on cancer risk but found that supplementation with $\beta$-carotene can increase cancer risk and mortality among smokers ${ }^{(57)}$. Of the observational studies that have examined any supplement use and $\mathrm{EC}^{(5,6,28,30,58)}$, one showed a reduced risk with B-only vitamins and a reduced risk with multivitamin use that was further decreased with increasing duration of use $^{(30)}$. Another study found a reduced risk with $\mathrm{Ca}$ and an increased risk with Fe supplementation ${ }^{(58)}$. The Women's Health Initiative concluded that there was no significant association of EC with multivitamin use; however, there was a marginal, but non-significant, increased risk with longer duration of use ${ }^{(59)}$.

Only two studies have examined the risk of EC in relation to micronutrient intake from food and supplements combined $^{(5,6)}$. Jain et al. $^{(5,6)}$ found no association with vitamin C intake in a case-cohort analysis ${ }^{(6)}$ and found a significant reduced risk with vitamin $\mathrm{E}(\mathrm{OR}=0 \cdot 61,95 \% \mathrm{CI} 0 \cdot 43,0 \cdot 88)$ 
in a case-control study that examined vitamins C, E and A and $\beta$-carotene from food plus supplements ${ }^{(5)}$.

We found a statistically significant $28-38 \%$ reduction in EC risk with $\mathrm{Ca}$ intake when dietary and supplemental sources were combined. Dietary $\mathrm{Ca}$ intake has been assessed in four previous hospital-based case-control studies $^{(25,28,34,52)}$, and supplemental Ca has been assessed in one hospital-based ${ }^{(28)}$ and one population-based case-control study ${ }^{(58)}$. Dietary Ca reduced EC risk by $52-61 \%$ in two studies ${ }^{(25,34)}$ and Ca supplements reduced risk by $50 \%$, which became $70 \%$ among women whose dairy intake was below the median ${ }^{(58)}$. The other study reported a non-significant reduced risk with ever use ${ }^{(28)}$. Because of a lack of intervention and cohort studies of $\mathrm{Ca}$ intake, and because of high heterogeneity between estimates, a review of these studies concluded that evidence to date is too sparse and inconclusive ${ }^{(60)}$. Our study supports the hypothesis that $\mathrm{Ca}$ from food and from supplementation lowers EC risk. The strengthened reductions in risk that we observed in our analysis restricted to non-users of supplemental Ca suggest that dietary $\mathrm{Ca}$ is important in EC risk reduction, which may reflect better dietary $\mathrm{Ca}$ absorption. Dietary $\mathrm{Ca}$, especially from dairy foods, is important in energy metabolism and can help maintain a healthy body weight ${ }^{(61)}$. Greater weight and fat loss have been observed in randomized trials for groups with high-dairy supplementation ${ }^{(62-65)}$. These findings are still inconclusive, however, as a review of randomized controlled trials of $\mathrm{Ca}$ supplementation and body weight revealed no association ${ }^{(66)}$. Ca supplementation may be beneficial only in populations with low baseline $\mathrm{Ca}$ intake ${ }^{(67)}$. For cancer prevention, Ca may act through vitamin D, a nutrient with anti-neoplastic potential, as it is highly correlated and metabolically tied to vitamin D. Vitamin D plays a role in cellular growth and differentiation through vitamin D receptors that are present in endometrial tissue ${ }^{(68,69)}$. Our analysis did not reveal an association with dietary intake of vitamin D; however, circulating serum levels of 25-hydroxyvitamin D may be more relevant in cancer prevention since a substantial portion of vitamin D is derived from the sun. A recent nested case-control study of 830 cases and 992 controls from seven cohorts, however, found no association between circulating 25 -hydroxyvitamin $\mathrm{D}$ and $\mathrm{EC}^{(70)}$.

We observed a reduced risk within the second quartile of riboflavin intake in our study, suggesting that riboflavin may play a role in EC risk reduction. Riboflavin is being investigated in cancer aetiology because of its role in folate metabolism $^{(71)}$. Minimum adult requirements are between 0.5 and $0.8 \mathrm{mg} / \mathrm{d}$ and urinary excretion increases at intakes of $1 \cdot 1-1.6 \mathrm{mg} / \mathrm{d}^{(45)}$. Since risk was not reduced at higher levels in our study, our results would be consistent with riboflavin bioavailability, suggesting that adequate levels may provide benefit. We observed unexpected increased risks with dietary soluble fibre, vitamin $C$, thiamin, vitamin $\mathrm{B}_{6}$ and lutein/zeaxanthin and with combined vitamin $\mathrm{B}_{12}$ intakes. Detection of risks at limited levels of intake for these nutrients, an absence of linear trend and the presence of multiple comparisons suggest that these may be chance findings. Our results for vitamins $\mathrm{B}_{6}$ and $\mathrm{B}_{12}$ might be reflective of meat intake, as higher intake of meat, particularly of red meat, has been associated with an increased risk for $\mathrm{EC}^{(47)}$. Adjustment for meat intake attenuated these associations only marginally, but there could be residual confounding. The increased risk we observed for vitamin C from diet is in contrast to the results from other stu$\operatorname{dies}^{(20,21,23,30,51,52)}$ and could suggest something about foods high in vitamin C. A hypothesis suggesting that grapefruit intake, through inhibition of the cytochrome P450 3A4 enzyme system, can affect oestrogen metabolism, leading to higher levels of circulating oestrogen and increased breast cancer risk, was examined in the Multiethnic Cohort Study ${ }^{(72)}$. We were unable to examine grapefruit intake with respect to the observed association with vitamin $\mathrm{C}$ because it was not assessed separately from intake of other citrus fruits in our questionnaire.

Although our assessments of associations among nonusers of the given supplemental nutrient were limited by sample size, restriction caused the detected association to be attenuated towards the null for thiamin and vitamin $\mathrm{B}_{6}$ and it lost statistical significance for vitamin $\mathrm{C}$. Risk was also attenuated and became non-significant for lutein/zeaxanthin among non-users of any supplements. Meanwhile, the results for dietary Ca were strengthened with restriction to non-users of supplemental Ca. Our results highlight that misclassification on exposure and possible confounding by supplement use are issues in studies of dietary nutrients and disease risk, leading to potentially spurious associations when these additional nutrients are not accounted for. Restriction to non-users of given supplements in the analysis may provide additional insight into detected associations. Future studies should incorporate measures of supplement use in addition to dietary assessment.

The population-based design, large sample size and comprehensive information on a wide range of risk factors are the strengths of our study. We were also able to evaluate the influence of supplement use combined with dietary sources of micronutrients, which has been carried out in only a limited number of previous studies ${ }^{(5,6)}$. Limitations in dietary assessment are recognized, as is the possibility of recall bias because of the retrospective dietary assessment. Any random measurement error associated with reporting past dietary exposures tends to attenuate associations ${ }^{(73)}$. Nevertheless, we were able to detect statistically significant associations, with evidence for linear trend, for some of these dietary and supplemental exposures; however, our estimates are likely to be conservative. The supplemental exposure variables from our questionnaire summarized exposure from all forms of supplements; hence, we could not evaluate risk for nutrients derived from multivitamins in relation to individual vitamins. Further, the potential for confounding by other nutrients in multivitamins also exists. Another limitation was our low control response rate, which 
we addressed by comparing our sample to a larger sample of Alberta women from the Canadian Community Health Survey Cycle $2 \cdot 2$ to evaluate possible selection bias. From this comparison we found that the control group was somewhat more educated than the base population but was otherwise similar with regard to age, height, weight and $\mathrm{BMI}^{(7)}$.

In summary, our study is supportive of a positive association between dietary cholesterol and EC risk. Risk was reduced with intake of $\mathrm{Ca}$ from food sources and from food plus supplements. Prospective studies of detailed dietary intakes of nutrients from food and supplements and biomarkers of nutritional and metabolic status are needed to confirm the roles of overall diet and specific nutrients in $\mathrm{EC}$ risk and provide additional insight into mechanisms that may underlie a true impact.

\section{Acknowledgements}

The case-control study of endometrial cancer was conducted at the Tom Baker Cancer Centre in Calgary, Alberta, and was supported by grants from the National Cancer Institute of Canada with funding from the Canadian Cancer Society. C.M.F. was supported by a Career Award from the Canadian Institutes of Health Research; C.M.F. and L.S.C. were supported by career awards from the Alberta Heritage Foundation for Medical Research; and L.S.C. and K.S.C. were supported by Canada Research Chairs. The authors have no conflict of interest to declare. R.K.B. conceptualized the study, performed the analysis and wrote the manuscript; C.M.F. and I.C. provided expertise in nutritional epidemiology; C.M.F., L.S.C., K.S.C. and A.M.M. obtained funding for data collection. All authors reviewed and contributed to drafts of the manuscript.

\section{References}

1. Canadian Cancer Society's Steering Committee (2010) Canadian Cancer Statistics 2010. Toronto: Canadian Cancer Society.

2. Kaaks R, Lukanova A \& Kurzer MS (2002) Obesity, endogenous hormones, and endometrial cancer risk: a synthetic review. Cancer Epidemiol Biomarkers Prev 11, 1531-1543.

3. World Cancer Research Fund/American Institute for Cancer Research (2007) Food, Nutrition, Physical Activity, and the Prevention of Cancer: a Global Perspective. Washington, DC: AICR.

4. Vatanparast H, Adolphe JL \& Whiting SJ (2010) Socioeconomic status and vitamin/mineral supplement use in Canada. Health Rep 21, issue 4, 19-25.

5. Jain MG, Howe GR \& Rohan TE (2000) Nutritional factors and endometrial cancer in Ontario, Canada. Cancer Control 7, 288-296.

6. Jain MG, Rohan TE, Howe GR et al. (2000) A cohort study of nutritional factors and endometrial cancer. Eur $J$ Epidemiol 16, 899-905.

7. Friedenreich CM, Cook LS, Magliocco AM et al. (2010) Case-control study of lifetime total physical activity and endometrial cancer risk. Cancer Causes Control 21, 1105-1116.
8. Waksberg J (1978) Sampling methods for random digit dialing. J Am Stat Soc 73, 40-46.

9. National Cancer Institute (2007) Diet History Questionnaire http://riskfactor.cancer.gov/DHQ/ (accessed June 2007).

10. Subar AF, Kipnis V, Troiano RP et al. (2003) Using intake biomarkers to evaluate the extent of dietary misreporting in a large sample of adults: the OPEN study. Am J Epidemiol 158, 1-13.

11. Subar AF, Thompson FE, Kipnis V et al. (2001) Comparative validation of the Block, Willett, and National Cancer Institute food frequency questionnaires: the Eating at America's Table Study. Am J Epidemiol 154, 1089-1099.

12. Thompson FE, Subar AF, Brown CC et al. (2002) Cognitive research enhances accuracy of food frequency questionnaire reports: results of an experimental validation study. J Am Diet Assoc 102, 212-225.

13. Barr SI, Kwan S \& Janelle KC (1994) Nutrient analysis using computer programs: comparison of a Canadian and an American database. J Am Diet Assoc 91, 29-32.

14. Csizmadi I, Kahle L, Ullman R et al. (2007) Adaptation and evaluation of the National Cancer Institute's Diet History Questionnaire and nutrient database for Canadian populations. Public Health Nutr 10, 88-96.

15. National Academy of Sciences, Institute of Medicine (1998) Dietary Reference Intakes for Thiamin, Riboflavin, Niacin, Vitamin B6, Folate, Vitamin B12, Pantothenic Acid, Biotin, and Choline. Washington, DC: National Academy Press.

16. Maruti SS, Ulrich CM \& White E (2009) Folate and one-carbon metabolism nutrients from supplements and diet in relation to breast cancer risk. Am J Clin Nutr 89, 624-633.

17. Willett WC, Howe GR \& Kushi LH (1997) Adjustment for total energy intake in epidemiologic studies. Am J Clin Nutr 65, 4 Suppl., 1220S-1228S.

18. Kleinbaum D \& Kupper L (1978) Applied Regression Analysis and Other Multivariable Methods. Belmont, CA: Wadsworth Publishing Company Inc.

19. Hosmer DW \& Lemeshow S (1989) Applied Logistic Regression. New York, NY: Wiley.

20. Goodman MT, Hankin JH, Wilkens LR et al. (1997) Diet, body size, physical activity, and the risk of endometrial cancer. Cancer Res 57, 5077-5085.

21. Goodman MT, Wilkens LR, Hankin JH et al. (1997) Association of soy and fiber consumption with the risk of endometrial cancer. Am J Epidemiol 146, 294-306.

22. Littman AJ, Beresford SA \& White E (2001) The association of dietary fat and plant foods with endometrial cancer (United States). Cancer Causes Control 12, 691-702.

23. McCann SE, Freudenheim JL, Marshall JR et al. (2000) Diet in the epidemiology of endometrial cancer in western New York (United States). Cancer Causes Control 11, 965-974.

24. Potischman N, Swanson CA, Brinton LA et al. (1993) Dietary associations in a case-control study of endometrial cancer. Cancer Causes Control 4, 239-250.

25. Salazar-Martinez E, Lazcano-Ponce E, Sanchez-Zamorano LM et al. (2005) Dietary factors and endometrial cancer risk. Results of a case-control study in Mexico. Int J Gynecol Cancer 15, 938-945.

26. Shu XO, Zheng W, Potischman $\mathrm{N}$ et al. (1993) A population-based case-control study of dietary factors and endometrial cancer in Shanghai, People's Republic of China. Am J Epidemiol 137, 155-165.

27. Potischman N, Hoover RN, Brinton LA et al. (1996) Case-control study of endogenous steroid hormones and endometrial cancer. J Natl Cancer Inst 88, 1127-1135.

28. Barbone F, Austin H \& Partridge EE (1993) Diet and endometrial cancer: a case-control study. Am J Epidemiol 137, 393-403.

29. Bandera EV, Kushi LH, Moore DF et al. (2007) Dietary lipids and endometrial cancer: the current epidemiologic evidence. Cancer Causes Control 18, 687-703. 
30. Xu WH, Dai Q, Xiang YB et al. (2007) Nutritional factors in relation to endometrial cancer: a report from a populationbased case-control study in Shanghai, China. Int J Cancer 120, 1776-1781.

31. Yeh M, Moysich KB, Jayaprakash V et al. (2009) Higher intakes of vegetables and vegetable-related nutrients are associated with lower endometrial cancer risks. J Nutr 139, 317-322.

32. Lucenteforte E, Talamini R, Montella M et al. (2008) Macronutrients, fatty acids and cholesterol intake and endometrial cancer. Ann Oncol 19, 168-172.

33. Prentice RL, Thomson CA, Caan B et al. (2007) Low-fat dietary pattern and cancer incidence in the Women's Health Initiative Dietary Modification Randomized Controlled Trial. J Natl Cancer Inst 99, 1534-1543.

34. Tzonou A, Lipworth L, Kalandidi A et al. (1996) Dietary factors and the risk of endometrial cancer: a case-control study in Greece. Br J Cancer 73, 1284-1290.

35. Hiatt RA \& Fireman BH (1986) Serum cholesterol and the incidence of cancer in a large cohort. J Chronic Dis 39, 861-870.

36. Kark JD, Smith AH \& Hames CG (1980) The relationship of serum cholesterol to the incidence of cancer in Evans County, Georgia. J Chronic Dis 33, 311-332.

37. Lindemann K, Vatten LJ, Ellstrom-Engh M et al. (2009) Serum lipids and endometrial cancer risk: results from the HUNT-II study. Int J Cancer 124, 2938-2941.

38. Swanson CA, Potischman N, Barrett RJ et al. (1994) Endometrial cancer risk in relation to serum lipids and lipoprotein levels. Cancer Epidemiol Biomarkers Prev 3, 575-581.

39. Wallace RB, Rost C, Burmeister LF et al. (1982) Cancer incidence in humans: relationship to plasma lipids and relative weight. J Natl Cancer Inst 68, 915-918.

40. Williams RR, Sorlie PD, Feinleib M et al. (1981) Cancer incidence by levels of cholesterol. JAMA 245, 247-252.

41. Zhang Y, Liu Z, Yu X et al. (2010) The association between metabolic abnormality and endometrial cancer: a large case-control study in China. Gynecol Oncol 117, 41-46.

42. Ye SQ \& Kwiterovich PO Jr (2000) Influence of genetic polymorphisms on responsiveness to dietary fat and cholesterol. Am J Clin Nutr 72, 5 Suppl., 1275S-1284S.

43. Willnow TE \& Nykjaer A (2010) Cellular uptake of steroid carrier proteins - mechanisms and implications. Mol Cell Endocrinol 316, 93-102.

44. Barrett-Connor E, Slone S, Greendale G et al. (1997) The Postmenopausal Estrogen/Progestin Interventions Study: primary outcomes in adherent women. Maturitas 27, 261-274.

45. The Nutrition Society (2002) Introduction to Human Nutrition. Oxford, UK: Blackwell Science Ltd.

46. Key TJ, Allen NE, Verkasalo PK et al. (2001) Energy balance and cancer: the role of sex hormones. Proc Nutr Soc 60, 81-89.

47. Bandera EV, Kushi LH, Moore DF et al. (2007) Consumption of animal foods and endometrial cancer risk: a systematic literature review and meta-analysis. Cancer Causes Control 18, 967-988.

48. Kabat GC, Miller AB, Jain M et al. (2008) Dietary iron and haem iron intake and risk of endometrial cancer: a prospective cohort study. Br J Cancer 98, 194-198.

49. Kallianpur AR, Lee SA, Xu WH et al. (2010) Dietary iron intake and risk of endometrial cancer: a population-based casecontrol study in Shanghai, China. Nutr Cancer 62, 40-50.

50. La Vecchia C, Decarli A, Fasoli M et al. (1986) Nutrition and diet in the etiology of endometrial cancer. Cancer 57, $1248-1253$.

51. Levi F, Franceschi S, Negri E et al. (1993) Dietary factors and the risk of endometrial cancer. Cancer 71, 3575-3581.

52. Negri E, La VC, Franceschi S et al. (1996) Intake of selected micronutrients and the risk of endometrial carcinoma. Cancer 77, 917-923.
53. Pelucchi C, Dal ML, Montella M et al. (2008) Dietary intake of carotenoids and retinol and endometrial cancer risk in an Italian case-control study. Cancer Causes Control 19, 1209-1215.

54. Salazar-Martinez E, Lazcano-Ponce EC, Lira-Lira GG et al. (2000) Case-control study of diabetes, obesity, physical activity and risk of endometrial cancer among Mexican women. Cancer Causes Control 11, 707-711.

55. Bandera EV, Gifkins DM, Moore DF et al. (2009) Antioxidant vitamins and the risk of endometrial cancer: a dose-response meta-analysis. Cancer Causes Control 20, 699-711.

56. Jeong NH, Song ES, Lee JM et al. (2009) Preoperative levels of plasma micronutrients are related to endometrial cancer risk. Acta Obstet Gynecol Scand 88, 434-439.

57. Bardia A, Tleyjeh IM, Cerhan JR et al. (2008) Efficacy of antioxidant supplementation in reducing primary cancer incidence and mortality: systematic review and metaanalysis. Mayo Clin Proc 83, 23-34.

58. Terry P, Vainio H, Wolk A et al. (2002) Dietary factors in relation to endometrial cancer: a nationwide case-control study in Sweden. Nutr Cancer 42, 25-32.

59. Neuhouser ML, Wassertheil-Smoller S, Thomson C et al. (2009) Multivitamin use and risk of cancer and cardiovascular disease in the Women's Health Initiative cohorts. Arch Intern Med 169, 294-304.

60. McCullough ML, Bandera EV, Moore DF et al. (2008) Vitamin $\mathrm{D}$ and calcium intake in relation to risk of endometrial cancer: a systematic review of the literature. Prev Med 46, 298-302.

61. Major GC, Chaput JP, Ledoux M et al. (2008) Recent developments in calcium-related obesity research. Obes Rev 9, 428-445.

62. Zemel MB, Thompson W, Milstead A et al. (2004) Calcium and dairy acceleration of weight and fat loss during energy restriction in obese adults. Obes Res 12, 582-590.

63. Zemel MB, Richards J, Mathis S et al. (2005) Dairy augmentation of total and central fat loss in obese subjects. Int J Obes (Lond) 29, 391-397.

64. Zemel MB, Richards J, Milstead A et al. (2005) Effects of calcium and dairy on body composition and weight loss in African-American adults. Obes Res 13, 1218-1225.

65. Eagan MS, Lyle RM, Gunther CW et al. (2006) Effect of 1-year dairy product intervention on fat mass in young women: 6-month follow-up. Obesity (Silver Spring) 14, 2242-2248.

66. Trowman R, Dumville JC, Hahn S et al. (2006) A systematic review of the effects of calcium supplementation on body weight. Br J Nutr 95, 1033-1038.

67. Gibson TM, Ferrucci LM, Tangrea JA et al. (2010) Epidemiological and clinical studies of nutrition. Semin Oncol 37, 282-296.

68. Vigano P, Lattuada D, Mangioni S et al. (2006) Cycling and early pregnant endometrium as a site of regulated expression of the vitamin D system. J Mol Endocrinol 36, 415-424.

69. Vienonen A, Miettinen S, Blauer M et al. (2004) Expression of nuclear receptors and cofactors in human endometrium and myometrium. J Soc Gynecol Investig 11, 104-112.

70. Zeleniuch-Jacquotte A, Gallicchio L, Hartmuller V et al. (2010) Circulating 25-hydroxyvitamin D and risk of endometrial cancer: Cohort Consortium Vitamin D Pooling Project of Rarer Cancers. Am J Epidemiol 172, 36-46.

71. Powers HJ (2003) Riboflavin (vitamin B-2) and health. Am J Clin Nutr 77, 1352-1360.

72. Monroe KR, Murphy SP, Kolonel LN et al. (2007) Prospective study of grapefruit intake and risk of breast cancer in postmenopausal women: the Multiethnic Cohort Study. Br J Cancer 97, 440-445.

73. Kipnis V \& Freedman LS (2008) Impact of exposure measurement error in nutritional epidemiology. $J$ Natl Cancer Inst 100, 1658-1659. 